\title{
Infliximah-induced erythema multiforme in a patient with chronic sarcoidosis
}

\section{Dear Editor}

Tumor Necrosis Factor- $\alpha$ (TNF- $\alpha$ ) inhibitors, in particular infliximab, have shown effectiveness as a third-line treatment option in relapsing, refractory sarcoidosis that requires an increased dose of corticosteroids plus one or more anti-sarcoidosis disease modifying drugs [1]. Infliximab has demonstrated effectiveness in severe pulmonary sarcoidosis and in extra-pulmonary sarcoidosis with multi-organ involvement. Bone involvement is reported to occur in $5-13 \%$ of patients at some point during their chronic disease course. Recently, in a study of 64 sarcoidosis patients, Zhou et al. found that bone disease occurs mainly in white females with chronic multi-organ disease. This bone involvement most commonly affects the spine (68.8\%), pelvis (35.9\%), and hands (15.6\%). These patients are chronically treated with corticosteroids and almost a quarter of them $(23.5 \%)$ received infliximab, which appears to be beneficial in chronic bone sarcoidosis [2].

In this article, we report on a case of a 68-yearold Caucasian female with chronic active sarcoidosis which had pulmonary, cutaneous, and joint involvement. The patient also had an unbalanced calcium metabolism. Physical examination of the hands exhibited severe dactylitis (purple-violet discoloration, swelling, distortion, pain, numbness, and nail dystrophy). Due to the relapsing nature of her disease, significant arthralgia after the tapering of pharmacological therapy (prednisone below $15 \mathrm{mg}$ in combination with leflunomide $20 \mathrm{mg} / \mathrm{d}$ ), and significant side-effects (osteoporo- sis), she was started on infliximab $5 \mathrm{mg} / \mathrm{kg}$. She received 6 doses of infliximab during a 6 month period (at 0, 2, 6, 12, 18, and 24 weeks, respectively). At the end of the 6 months of treatment, the patient showed significant clinical improvement with complete resolution of her arthritis. Angiotensin-converting enzyme levels fell from $110 \mathrm{U} / \mathrm{l}$ to $58 \mathrm{U} / \mathrm{l}$. However, shortly before the last infliximab infusion, she developed an erythematous, patchy, and painful cutaneous lesion located unilaterally and affecting the dorsal, lateral, and phalangeal skin surface of the right lower foot (Figure 1A). The lesion was biopsied and a histopathological examination revealed erythema multiforme (EM) (Figure 1B). Since the patient had not received any other medications which had been known to cause EM, and all cultures and tests for viruses, fungi, and microbes were negative, this was considered to be an infliximab-related infusion reaction resulting in erythema multiforme. After the $6^{\text {th }}$ infusion, infliximab was withdrawn (as initially planned) and EM gradually subsided without any other intervention.

In two recently published studies focusing on refractory sarcoidosis patients with cutaneous symptoms, French investigators treating these patients with anti-TNF- $\alpha$ agents reported discontinuation rates due to adverse effects of $24 \%$ and $23 \%$, respectively [3, 4]. Notable side effects that necessitated withdrawing treatment with anti-TNF- $\alpha$ agents included infections and serious immunological reactions. On the other hand, cutaneous reactions stemming from the use of these agents are described to occur commonly (up to

Address for correspondence: llias Papanikolaou, Corfu General Hospital, Pulmonary Department, Corfu, Greece; e-mail: icpapanikolaou@hotmail.com DOI: 10.5603/ARM.2020.0131

Received: 16.04 .2020

Copyright (C) 2020 PTChP

ISSN 2451-4934 

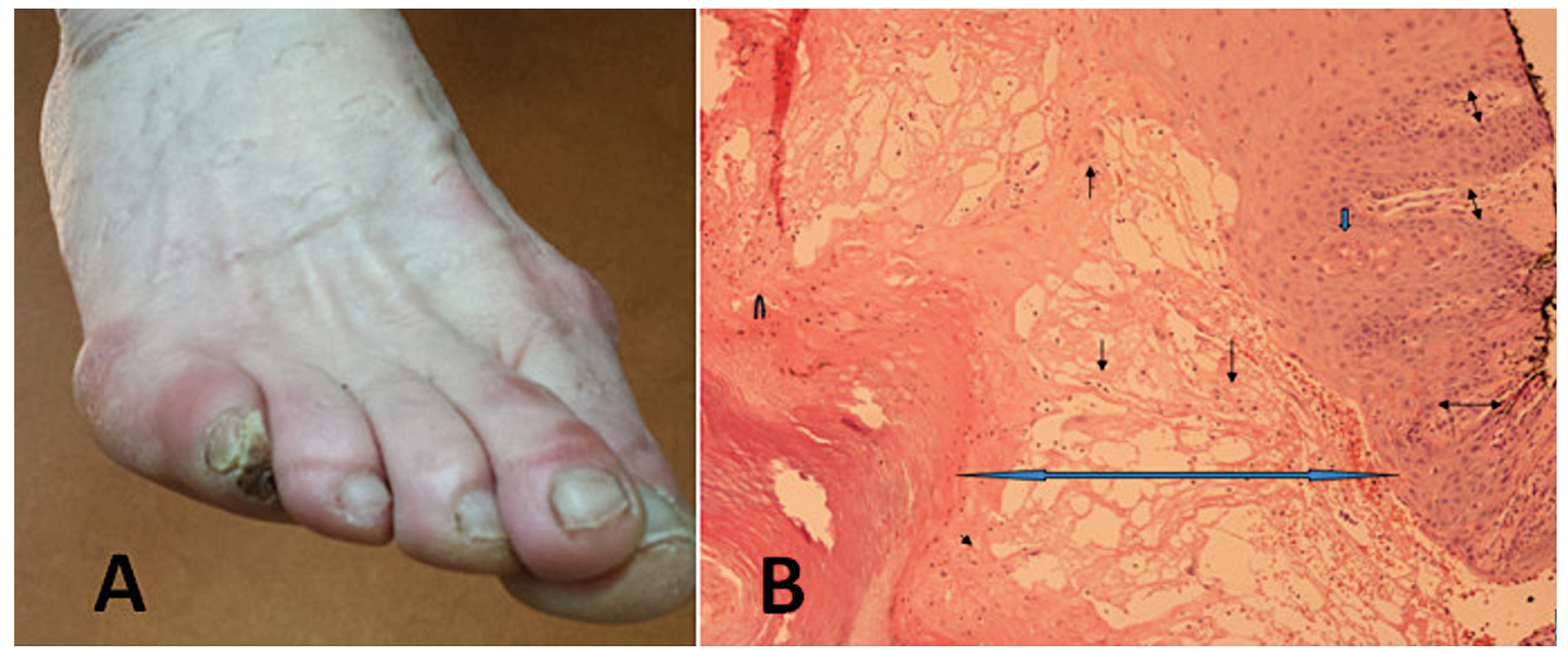

Figure 1. A. Patchy erythematous lesions of the right lower foot at the resolution phase after infliximab discontinuation. B. Erythema multiforme: Intraepidermal edema with liquefactive necrosis (double thick arrow), lymphocytic exocytosis (simple arrows), spongiosis (simple thick arrow), dyskeratotic cells, and dermal inflammatory infiltrate including lymphocytes and histiocytes (double thin arrow) (Hematoxylin-Eosin stain X100)

$25 \%)$ in patients treated for rheumatologic conditions and inflammatory bowel diseases. Such reactions include psoriasis, xerosis, eczema, skin infections, skin cancer, palmoplantar pustulosis, alopecia areata, bullous pemphigoid, drug-induced lupus, and urticaria [5, 6]. However, EM due to infliximab is rare. Although a 2009 Food and Drug Administration (FDA) report included infliximab cutaneous adverse events, only 21 such cases have been reported and all were related to rheumatologic conditions $[7,8]$. This case is the first to our knowledge to describe EM after therapy with infliximab in a patient with sarcoidosis.

Cutaneous involvement in sarcoidosis is frequent, affecting up to $25 \%$ of patients at some point in their disease course. In our patient, the differential diagnosis included specific or non-specific cutaneous sarcoidosis involvement, skin infections, drug-related reactions, and paradoxical sarcoid-like reactions (reported mainly with use of etanercept in rheumatoid arthritis and inflammatory bowel diseases) [9]. The updated ACCESS organ assessment tool classified our patient`s skin lesions as possibly being due to sarcoidosis because of their atypical nature, therefore, histologic confirmation was mandatory [10]. Since no granulomas were discovered and a temporal association with infliximab administration existed, we considered this EM to be infliximab-related.

Infliximab-induced EM may warrant drug interruption or discontinuation. Therefore, it is important to consider infliximab-induced EM in the differential diagnosis. This would have significant implications for the patient because it could lead to avoiding unnecessary treatment escalation for presumed treatment failure and allowing for appropriate local or systemic EM therapy as well as, if necessary, changing the treatment. In our case, tapering infliximab resulted in EM resolution. Corticosteroids (prednisone) were successfully tapered to a dose of $7.5 \mathrm{mg}$. The patient also received leflunomide. The patient has remained stable for 18 months without the need to introduce another mode of pharmacological treatment.

\section{References:}

1. Baughman RP, Drent M, Kavuru M, et al. Sarcoidosis Investigators. Infliximab therapy in patients with chronic sarcoidosis and pulmonary involvement. Am J Respir Crit Care Med. 2006; 174(7): 795-802, doi: 10.1164/rccm.200603-402OC, indexed in Pubmed: 16840744

2. Zhou Y, Lower EE, Li H, et al. Clinical characteristics of patients with bone sarcoidosis. Semin Arthritis Rheum. 2017; 47(1): 143-148, doi: 10.1016/j.semarthrit.2017.02.004, indexed in Pubmed: 28274482.

3. Heidelberger V, Ingen-Housz-Oro S, Marquet A, et al. Efficacy and tolerance of anti-tumor necrosis factor $\alpha$ agents in cutaneous sarcoidosis: a french study of 46 cases. JAMA Dermatol. 2017; 153(7): 681-685, doi: 10.1001/jamadermatol.2017.1162, indexed in Pubmed: 28564695.

4. Jamilloux Y, Cohen-Aubart F, Chapelon-Abric C, et al. Groupe Sarcoïdose Francophone. Efficacy and safety of tumor necrosis factor antagonists in refractory sarcoidosis: A multicenter study of 132 patients. Semin Arthritis Rheum. 2017; 47(2): 288-294, doi: 10.1016/j.semarthrit.2017.03.005, indexed in Pubmed: 28392046.

5. Havmose M, Thomsen SF. Development of paradoxical inflammatory disorders during treatment of psoriasis with TNF inhibitors: a review of published cases. Int J Dermatol. 2017 56(11): 1087-1102, doi: 10.1111/ijd.13691, indexed in Pubmed: 28737221 
6. Segaert S, Hermans C. Clinical signs, pathophysiology and management of cutaneous side effects of anti-tumor necrosis factor agents. Am J Clin Dermatol. 2017; 18(6): 771-787, doi: 10.1007/s40257-017-0296-7, indexed in Pubmed: 28597181.

7. STN: BL 103772/5258. Food and Drug Administration report. 0ctober. ; 29: 2009

8. Edwards D, Boritz E, Cowen EW, et al. Erythema multiforme major following treatment with infliximab. Oral Surg Oral Med Oral Pathol Oral Radiol. 2013; 115(2): e36-e40, doi: 10.1016/j. oooo.2012.08.001, indexed in Pubmed: 23036796.
9. Decock A, Van Assche G, Vermeire S, et al. Sarcoidosis-Like lesions: another paradoxical reaction to anti-tnf therapy? J Crohns Colitis. 2017; 11(3): 378-383, doi: 10.1093/ecco-jcc/ jjw155, indexed in Pubmed: 27591675.

10. Judson MA, Costabel U, Drent M, et al. The WASOG Sarcoidosis Organ Assessment Instrument: An update of a previous clinical tool. Sarcoidosis Vasc Diffuse Lung Dis. 2014; 31(1): 19-27, indexed in Pubmed: 24751450. 